\title{
Aetiology and repair of tracheal stenosis following tracheostomy and intermittent positive pressure respiration
}

\author{
P. GIB SON \\ From the Thoracic Surgery Unit, Royal Perth Hospital, Perth, Western Australia
}

At Christmas time a few years ago a young man sustained a severely crushed chest in a country motor vehicle accident and was transferred to the Royal Perth Hospital for further management. He had a flail chest wall, a right pneumothorax with extensive surgical emphysema, and during the 100-mile ambulance ride to hospital he regurgitated and inhaled much of the beer which had been in his stomach at the time of the accident. He was fat and a heavy smoker.

His gross respiratory insufficiency was managed at first by means of a tracheostomy with assisted ventilation from a Bird respirator, but his anoxic state was not relieved until he was fully curarized. Over the next 10 days great difficulty was experienced in keeping his airways clear of thick, encrusted blood-stained secretions. His tracheostomy wound and bronchial tree became grossly infected with Staphylococcus aureus and Pseudomonas pyocyanea, and one of the many chest radiographs taken during this time clearly shows the cuff of the tracheostomy tube distended with air beyond the width of the trachea. On the twelfth day a piece of hyaline cartilage was removed from the region of the cuff, and bronchoscopy showed deep circumferential ulceration of the mucous membrane in this area, with several bare tracheal rings visible in the floor of the ulcer. The secondary infection in the tracheostomy wound was treated with antibiotics. He was weaned off the respirator three weeks after the accident and soon discharged.

Three weeks later he was readmitted moribund from anoxia due to a tight stricture of the trachea for $4 \mathrm{~cm}$. below the previous stoma at the site of the original ulceration. Trauma from the cuff of the tracheostomy tube, followed by infection in the wound, had resulted in destruction of the full thickness of a segment of the trachea and its replacement within a month by a tight, fibrous stricture. He has now had a staged plastic recon- struction of the trachea and leads a perfectly normal life.

\section{TRACHEOSTOMY MANAGEMENT}

Since this patient was first admitted a small intensive care unit of three beds has been set up in the same ward as the thoracic surgery unit of the Royal Perth Hospital. Between April 1963 and May 1966, 154 patients with respiratory insufficiency have been treated by intermittent positive pressure respiration (I.P.P.R.) using a cuffed tracheostomy tube. Of necessity, much of the nursing is carried out by trainee staff under the close supervision of trained staff who are well aware of the dangers of incorrect management. The cuffed tubes are left in place routinely for 48 to 72 hours in the first instance and are changed within this time only if there is a special indication. After this period the tubes are changed each 24 or 48 hours until they are finally replaced by an uncuffed metal or plastic tube. The latter are changed once or twice a week until they are finally discarded. Tracheostomy wound dressings are changed as often as necessary, usually every four hours in the first few days. No use has been made of local antibiotics. Aspiration of secretions from the trachea is carried out using sterile soft rubber Jacques catheters connected to the suction tubing with a Y-piece so that the suction can be broken intermittently (Plum and Dunning, 1956). The cuffs are released every half hour and every time the patient requires the aspiration of secretions, and a chart is kept of cuff release and inflation. All parts of the Bird respirators are sterilized in formalin vapour, and only sterile distilled water is used in the humidifiers and nebulizers. In the first 18 months sterile sodium bicarbonate solution was often used to soften encrusted secretions, but this has been abandoned because humidification has become more efficient 
and also because it has been shown to damage the ciliated respiratory epithelium (Sara, 1965).

Antibiotics are used as far as possible on bacteriological indications, though most patients receive a broad-spectrum antibiotic until specific bacteriology is known. Cultures of the tracheal wound and secretions are taken frequently whenever clinical infection is apparent, and in most cases routine cultures are taken every two or three days.

A study of the 154 patients treated in this way suggests that trauma from the cuff of the tracheostomy tube and secondary infection of the tracheostomy wound are the two main factors responsible for the destruction of the full thickness of a segment of the tracheal wall with subsequent tracheal stenosis.

\section{RESULTS}

Of the 154 patients, 58 died, usually because of the original illness, though also because of secondary pulmonary infections. Full necropsies were available in 28 cases, and of these only three had a normal trachea. Two of the three had had the tracheostomy for less than 24 hours and the third for 11 days. In the latter case the tracheostomy wound and the sputum grew coliform organisms for eight days before death, but, despite this, at necropsy 'the trachea was remarkably normal in appearance'. All the remaining 25 cases had macroscopic evidence of damage to the trachea at necropsy, though in none was this the actual cause of death. Eight had acute tracheitis with gross reddening and oedema of the tracheal mucosa in the region of the stoma, the area where the cuff had been in contact with the mucosa, and in the trachea below this, sometimes even extending into the main bronchi and sometimes associated with pneumonia. Often the mucosa was covered with much sticky blood-stained secretion. The remaining 17 cases all had similar tracheitis but with ulceration of the tracheal mucosa in the region of contact with the cuff, so that the cartilaginous rings were laid bare or even fragmented (Fig. 1), and in one case infected by Ps. pyocyanea small abscesses had formed in the wall of the trachea. Sometimes there were also smaller areas of ulceration below the cuff, particularly near the carina, suggesting trauma from the aspirating catheters.

Of the 25 cases with tracheal lesions at necropsy, two had sterile cultures from the tracheostomy wound during life and in eight the wounds were not cultured. The remaining 15 all

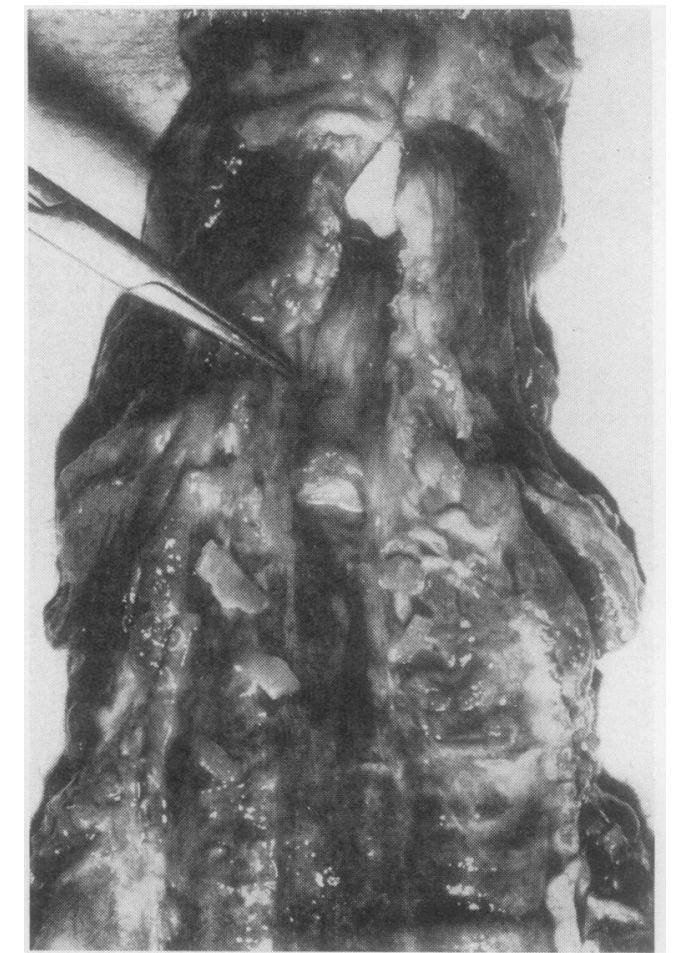

FIG. 1. Trachea opened posteriorly to show stoma, and destruction of the tracheal wall with fragmentation of tracheal rings.

had positive wound cultures, and the organisms most commonly grown were coliforms, $P$ s. 응 pyocyanea, Staph. aureus, and Friedländer's $\times$ bacillus (see Table I). In one patient who had Staph. aureus in the sputum before operation, superficial ulceration of the tracheal mucosa was seen less than 24 hours after tracheostomy.

Of the 154 patients comprising the whole series, 을 at least 95 had secondary infections in the $\frac{D}{2}$ tracheostomy wounds. However, in 47 cases the

T A B L E I

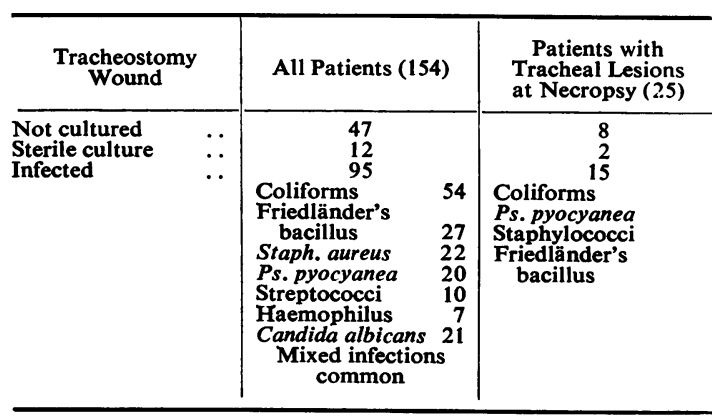


wounds were not cultured. In the remaining 12 cases the cultures were sterile (Table I). Coliforms were cultured from 54 patients, Friedländer's bacillus from 27, Staph. aureus from 22, Ps. pyocyanea from 20 , and other organisms, including streptococci, haemophilus, and Candida albicans, from 38 patients. Mixed infections were common.

During life the findings which were so common at post-mortem examination were not often observed, probably because there was no particular indication to look for them. Of the 96 patients who survived, only 10 were known to have tracheal ulceration or tracheitis while having I.P.P.R. All 10 of these had infected tracheostomy wounds and later developed some degree of tracheal stenosis proved by bronchoscopy or tomography, though so far only four have required treatment for the relief of symptoms.

In the present series the frequency with which the cuff was over-distended with air was not determined, for it was seldom recorded in the case notes. Since the cuff is often inflated and deflated, and since each patient is attended by many different nurses and doctors during his illness, it is possible that many will have the cuff overdistended at some stage.

The chest radiographs of 125 of the 154 patients were available, and a review of them showed that in 23 patients the cuff of the tracheostomy tube had been distended with air beyond the limits of the trachea at some time during the period of I.P.P.R. (Fig. 2). Fifteen of these patients survived, and six developed tracheal stenosis, three of whom required tracheal reconstruction (see Table II).

T A B L E I I

OVER-DISTENSION OF CUFF: CHEST RADIOGRAPHS OF 125 PATIENTS REVIEWED

\begin{tabular}{|c|c|c|c|c|}
\hline Over-distension & No. & Survived & Stenosis & $\begin{array}{l}\text { Required } \\
\text { Recon- } \\
\text { struction }\end{array}$ \\
\hline $\begin{array}{l}\text { Observed } \\
\text { Not observed }\end{array}$ & $\begin{array}{r}23 \\
102\end{array}$ & $\begin{array}{l}15 \\
62\end{array}$ & $\begin{array}{l}6 \\
4\end{array}$ & $\begin{array}{l}3 \\
1\end{array}$ \\
\hline
\end{tabular}

In the 102 patients in whom there was no radiographic evidence of over-distension of the cuff. 62 survived and only four developed tracheal stenosis, one of whom required tracheal reconstruction (Table II).

Four of the patients who developed tracheal stenosis (Fig. 3) had severe symptoms and required operation to relieve the obstruction. Two of these had tubular stenosis at the site of the cuff; one had two strictures, a tubular one at the cuff site and

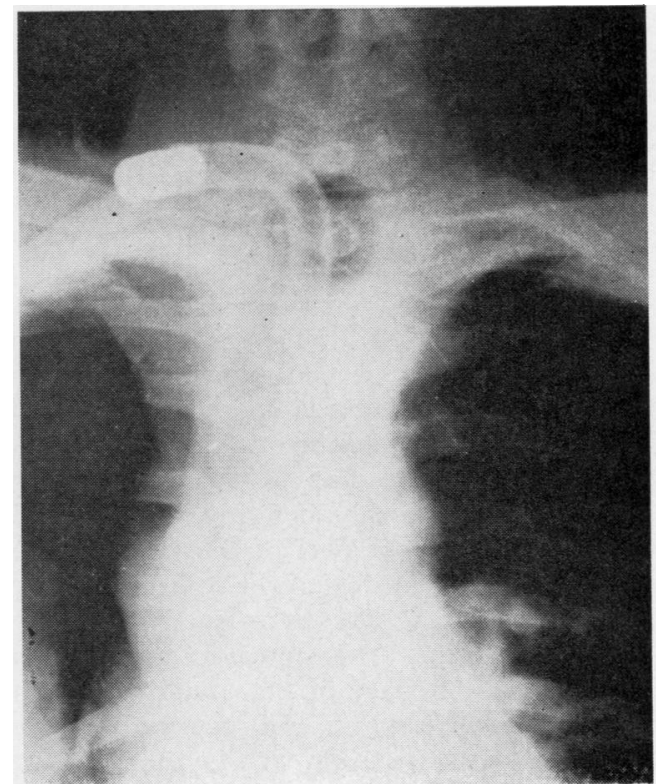

FIG. 2. Shows cuff of the tracheostomy tube distended with air beyond the limits of the trachea.

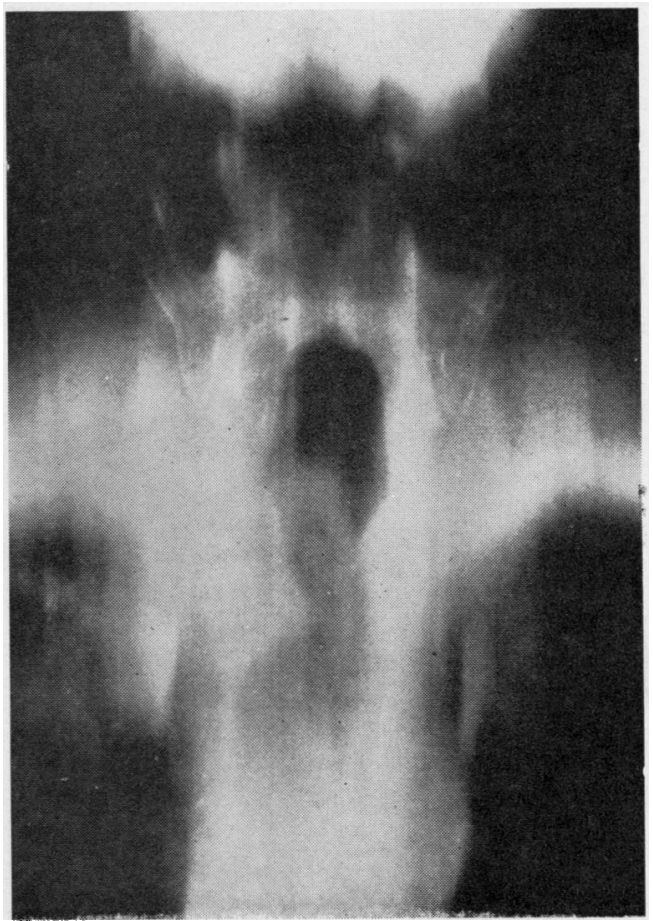

FIG. 3. Tomogram illustrating tracheal stricture about $3 \mathrm{~cm}$. long. 
a shorter one just above it at the site of the stoma ; and the fourth patient had a tubular stenosis at the level of the stoma and just below it. In each, the stricture was more than $2 \mathrm{~cm}$. long, precluding safe resection and end-to-end anastomosis (Rob and Bateman, 1949).

A staged plastic reconstruction was therefore devised (McComb, 1966). At the first stage, the stricture is divided longitudinally throughout its entire length and the incision is extended into normal trachea well below the lower limit of the stricture. The edges of the upper part of the guttered trachea are sutured to the edges of the vertical midline skin incision. At the same time a pectoral-based pedicle flap is swung from the anterior aspect of the shoulder (Fig. 4) and turned down behind the upper part of the sternum. The apex of the flap is sutured to the inferior margins of the incision in the trachea in such a way that the epithelium lines the anterior wall of the trachea. The raw surface of the shoulder and any unepithelialized areas of the guttered trachea are covered with split-skin grafts. A wide-bore tracheostomy tube is then worn for at least three months, and during this time any small posterior ridges of scar tissue are excised and grafted. When it is certain that all the remaining tracheal gutter is epithelialized, and that there is no tendency for the tracheal stoma to close in when the tracheostomy tube is discarded during a trial period of several weeks, the base of the pedicle is divided and turned up to form the anterior closure for the gutter. Lateral skin fiaps are then raised to cover the outer aspect of the graft.

Three of the patients treated in this way have had their operations completed and have had excellent relief of their symptoms. The fourth patient is still undergoing treatment.

\section{DISCUSSION}

Overinflation of the cuff of a tracheostomy tube may lead to pressure necrosis of the tracheal mucosa, particularly in patients with impaired circulation (Watts, 1963). Intermittent positive pressure respirators induce a piston-like motion in the tracheotomy tube, and it is postulated that this leads to shearing forces between the cuff and the tracheal mucosa (McComb, 1967). If this is so, even a correctly inflated cuff may inflict quite severe trauma on the mucosa. Once trauma to the tracheal mucosa has occurred, secondary infection is very likely to follow, and this may lead to destruction of the full thickness of a segment of the wall of the trachea.

Avoiding this secondary infection has proved to be the most difficult part of the management of patients treated by I.P.P.R. Often they require emergency aspiration of secretions under conditions which preclude both scrubbing up or successfully using a no-touch technique. Not infrequently the patient unwittingly infects his own tracheostomy; he may be incontinent of faeces one minute and clawing at his tracheostomy with a dirty hand the next. And even if not incontinent, how often does one see a patient with a tracheostomy, on I.P.P.R., offered a cake of soap and a bowl of water after defaecation into a bedpan ? It is more likely that he will obstruct the tracheostomy tube with his finger to ask if he can wash his hands.

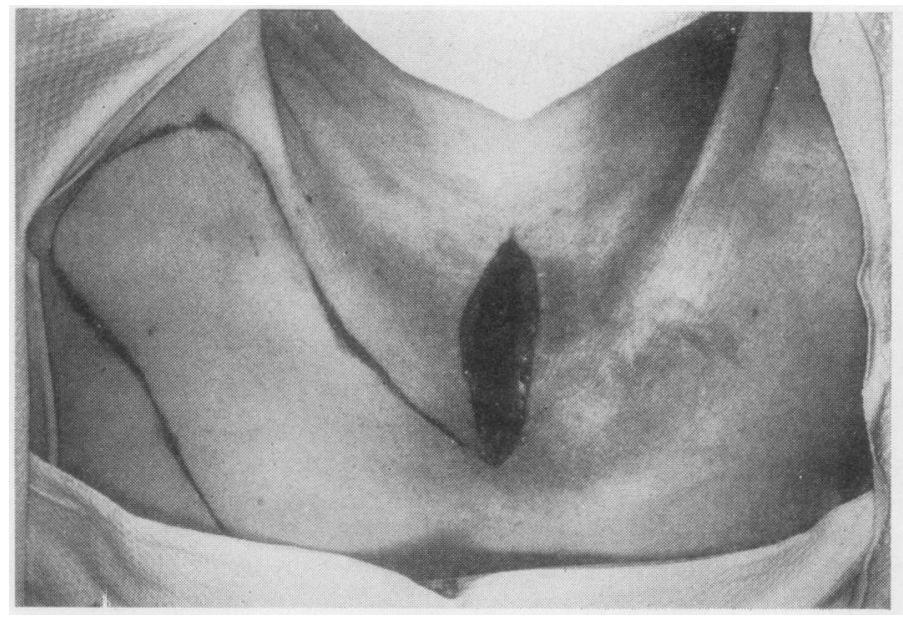

FIG. 4. Shows vertical incision through which the stricture is incised. The outline of the skin flap is marked. 


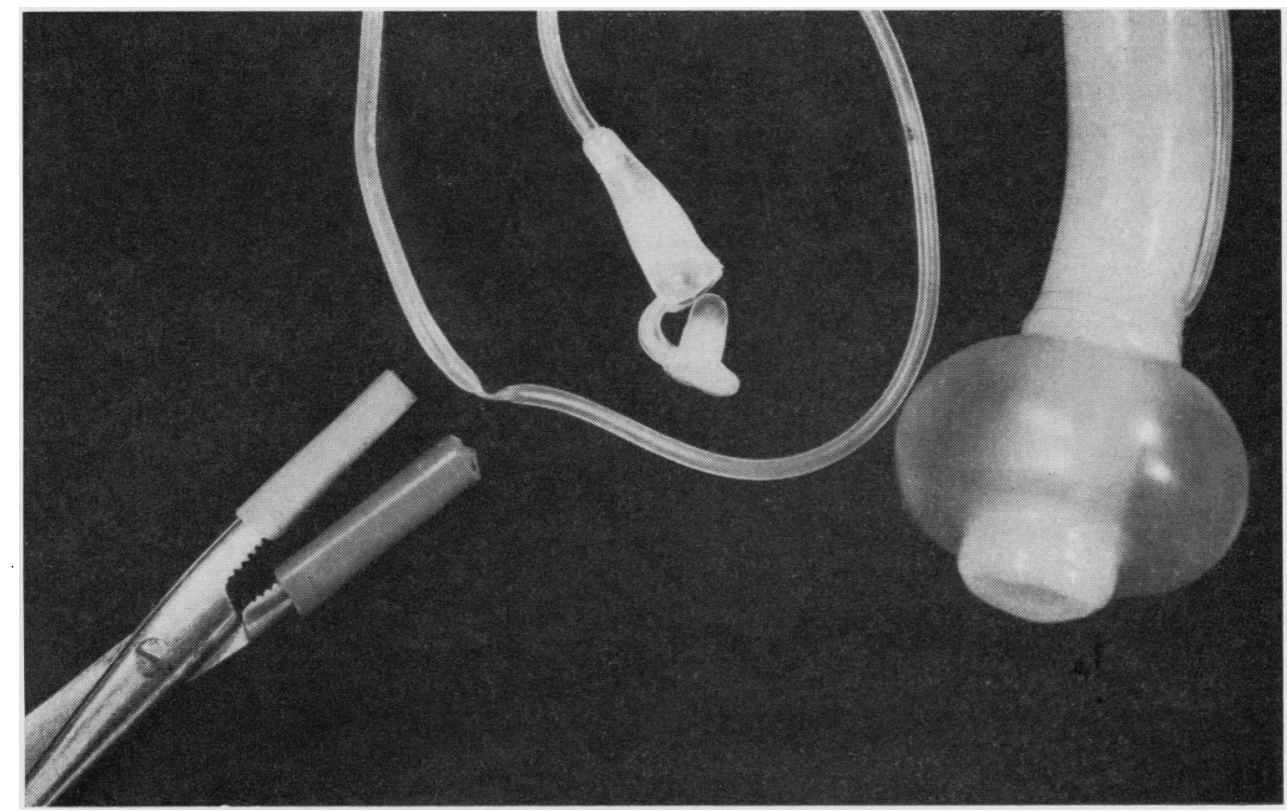

FIG. 5. The inflation tube remained occluded where it was clamped by the artery forceps, and the cuff remains tightly distended.

The management of the cuff also poses difficulties. It is important to have just the right amount of air in the cuff and no more, and to fix the tube so that a minimum of movement occurs. Frequent deflation of the cuff would seem to be essential, though opinions differ widely as to how often this should be done (Robertson, 1964 ; Kodicek, 1960 ; Watts, 1963 ; Glossop, 1966). Intermittent inflation of the cuff during inspiration may be achieved by means of a valve inserted in the pressure line of the Bird respirator (Cosby, 1965). However, this valve is not easy to adjust, and unless it can be redesigned we shall not use it. We have had no experience of long cuffs or of double cuffs, one above the other, though in theory both would be preferable to the single short cuffs of the standard Rusch tube (Kodicek, 1960 ; Glossop, 1966 ; Paloschi and Lynn, 1965). At one stage we used plastic cuffed tubes, but found that the nursing staff could not be broken of the habit of clamping the inflation tube with artery forceps. When this is done, the walls of the inflation tube are likely to remain occluded so that the cuff does not deflate when the clamp is removed (Fig. 5). These tubes have therefore been abandoned.

Once the tracheal wall is infected and destruction of its architecture begins, it yields to the pressure of the cuff and dilates, so that more and more air has to be put into the cuff to ensure an airtight seal. As shown in this study, the overdistended cuff can often be detected on the chest radiograph of those patients later found to have a higher incidence of serious stenosis. This radiological sign may well be only an indication of the severity of the infection rather than an indication of tracheal destruction by pressure.

\section{SUMMARY}

In the Intensive Care Unit at the Royal Perth Hospital 154 patients were treated by I.P.P.R. using a cuffed tracheostomy tube ; 96 patients survived.

In 28 necropsies the area of the trachea in contact with the cuff was ulcerated in 17 and was the site of an intense acute inflammation in another eight. Of these 25 cases, the tracheostomy wound was certainly infected in 15 .

In the 96 surviving patients, 10 were known during the period of I.P.P.R. to have tracheitis or ulceration of the trachea: they all had infected wounds and all developed tracheal stenosis of varying severity, four of them sufficiently severe to require operation. 
Review of the chest radiographs of 125 patients showed over-distension of the cuff in 23 , with 15 survivors, of whom six developed stenosis, including three with severe symptoms. In 62 survivors without obvious over-distension of the cuff, only four developed stenosis-one with severe symptoms.

In three patients with tracheal stenosis excellent results have been obtained with a staged plastic reconstruction of the trachea. A fourth patient is still undergoing treatment.

The difficulties of preventing secondary infection and of avoiding over-inflation of the cuff are briefly discussed.

\section{REFERENCES}

Cosby, W. M. (1965). Automatic intermittent inflation of tracheostomy-tube cuff, in Confidential Medical Abstracts, Folio 14. Commonwealth Industrial Gases Ltd., Alexandria, New South Wales.

Glossop, M. W. (1966). A cuffed tracheostomy tube. Lancet, 1, 187.

Kodicek, J. M. (1960). The place and management of tracheostomy $\mathbb{D}$ in respiratory insufficiency. $J$. Laryng., 74, 891.

McComb, H. (1967). Treatment of tracheal stenosis. To be published.

Paloschi, G., and Lynn, R. B. (1965). Observations upon elective and emergency tracheostomy. Surg. Gynec. Obstet., 120, 356.

Plum, F., and Dunning, M. F. (1956). Technics for minimizing trauma to the tracheobronchial tree after tracheotomy. New Engl. J. Med., 254, 193.

Rob, C. G., and Bateman, G. H. (1949). Reconstruction of the trachea and cervical oesophagus. Preliminary report. Brit. J. Surg., 37, 202.

Robertson, D. S. (1964). Tracheostomy and open heart surgery. N Proc, roy. Soc. Med., 57, 855.

Sara, C. (1965). The management of patients with a tracheostomy. Med. J. Aust., 1, 99.

Watts, J. McK. (1963). Tracheostomy in modern practice. Brit. J. 윽 Surg., 50, 954. 